\title{
Free Functional Gracilis Muscle Transfer for Reconstruction during Forearm Reimplantation in a Patient with Deep Venous Thrombosis
}

\author{
Daiki Morita, MD ${ }^{1}$ Hitoshi Nemoto, MD, $\mathrm{PhD}^{1} \quad$ Kenta Miyabe, $\mathrm{MD}^{1}$ Seiko Nakae, MD \\ Tomoaki Kuroki, MD, $\mathrm{PhD}^{1}$ Tomohiro Yasuda, MD, $\mathrm{PhD}^{2}$
} ${ }^{1}$ Department of Plastic and Reconstructive Surgery, Showa University
Fujigaoka Hospital, Yokohama, Japan
${ }^{2}$ Department of Orthopedic Surgery, Showa University Fujigaoka
Hospital, Yokohama, Japan
Address for correspondence Daiki Morita, MD, Department of Plastic and Reconstructive Surgery, Showa University Fujigaoka Hospital, 1-30, Fujigaoka Aobaku, Yokohama 227-8501, Japan (e-mail: d-morita@koto.kpu-m.ac.jp).

J Reconstr Microsurg Open 2018;3:e21-e24.

The forearm is frequently involved in extremity amputation. In cases of extremity amputation, treatment strategy includes considerations regarding the general condition of the patient, the site of the injury and severity of the tissue loss, functional prognosis, and social background.

Functional free muscle transfer is a valuable tool to reconstruct the upper extremity and is indicated for late reconstruction of brachial plexus injuries, traumatic muscle loss, Volkmann ischemic contracture, and loss resulting from oncologic resection. ${ }^{1,2}$ The gracilis muscle is one of the most commonly used donor muscles for functional muscle transfer, and is ideal for the reconstruction of the flexor group. ${ }^{1-4}$
Perioperative deep venous thrombosis (DVT) is a potentially fatal complication due to the risk of pulmonary embolism $(\mathrm{PE})^{5,6}$ There are several reports describing temporary inferior vena cava (IVC) filter placement preoperatively to prevent PE. ${ }^{7-9}$ However, standard perioperative management of DVT is unclear.

There are many factors that could lead to or exacerbate DVT/PE during free flap transfer, including the length of the operation, intraoperative position changes, and perioperative suspension of anticoagulants. We report our management strategy for a case of free gracilis muscle flap transfer for functional forearm reconstruction after traumatic amputation and reimplantation in a patient with a known DVT. received

December 30, 2017

accepted

February 22, 2018
DOI https://doi.org/

10.1055/s-0038-1642625. ISSN 2377-0813.
Copyright $\odot 2018$ by Thieme Medical Publishers, Inc., 333 Seventh Avenue, New York, NY 10001, USA. Tel: +1(212) 584-4662.
License terms

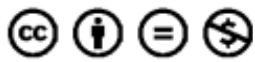



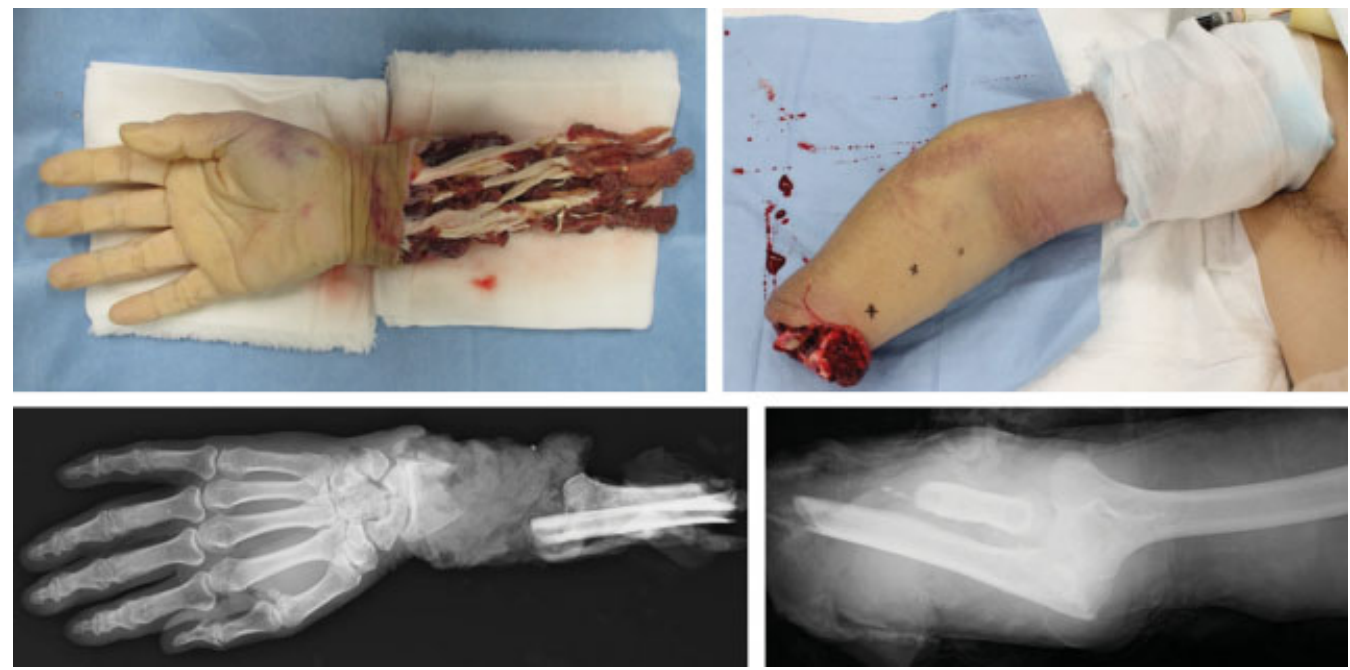

Fig. 1 Preoperative photographs and X-ray images. Right forearm with complete amputation with right elbow dislocation.

\section{Case Presentation}

A 62-year-old man suffered blunt amputation of the right forearm, right elbow dislocation, and hip fracture with dislocation after a stirring machine accident. He was brought by ambulance to our hospital ( - Fig. 1). Poor prognosis of his right arm in regard to function was predicted because his forearm was heavily crushed. Reimplantation of the amputated forearm was performed immediately after informed consent. First, the free bone fragments of the radius and ulna were fixed by orthopedic surgeons. Next, the radial and ulnar arteries were anastomosed using greater saphenous vein (GSV) conduit. The cephalic vein was reconstructed using interposition GSV conduit. While the median nerve could be directly repaired and the ulnar nerve repaired using a nerve regeneration-induced tube (Nerbridge, TOYOBO, Osaka, Japan), the radial nerve could not be repaired secondary to the length of the defect. Because the soft tissue defect was large and the bones could not be covered, an abdominal flap was harvested to cover the exposed bones, and artificial dermis (INTERGRA, Century Medical, Tokyo, Japan) was used to cover the exposed vessels (-Fig. 2). The reimplanted forearm almost completely survived. Although 10,000 units of heparin were injected daily for prophylaxis of DVT after reimplantation, D-dimer elevation was observed on postoperative day 20 , and computed tomography revealed a DVT
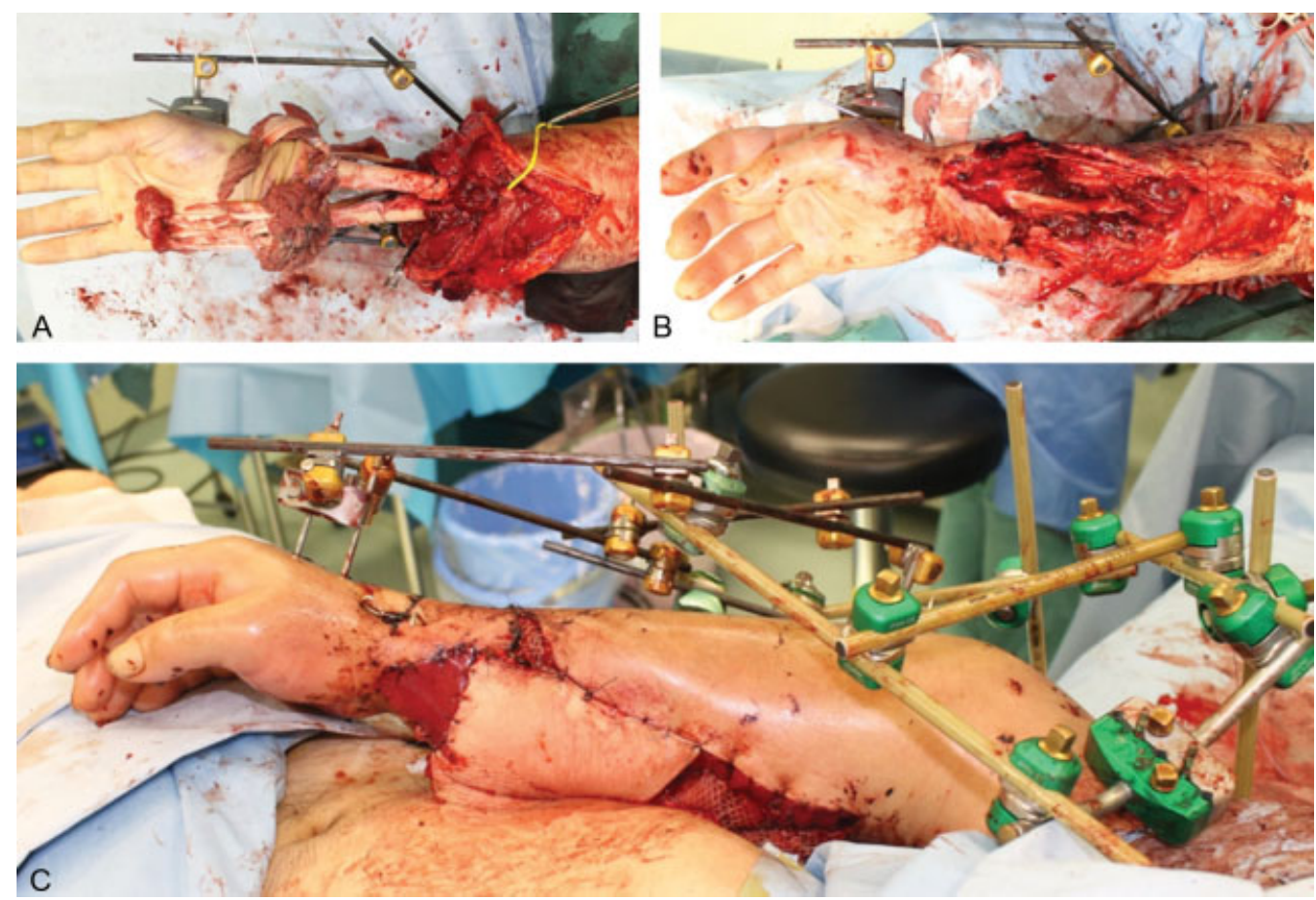

Fig. 2 Reimplantation operation. (A) Bony fixation using wires and external fixator. (B) Soft tissues were reimplanted. (C) Because the soft tissue defect was large, an abdominal flap was harvested to cover the exposed bone, and artificial dermis was used to cover the exposed vessels. 


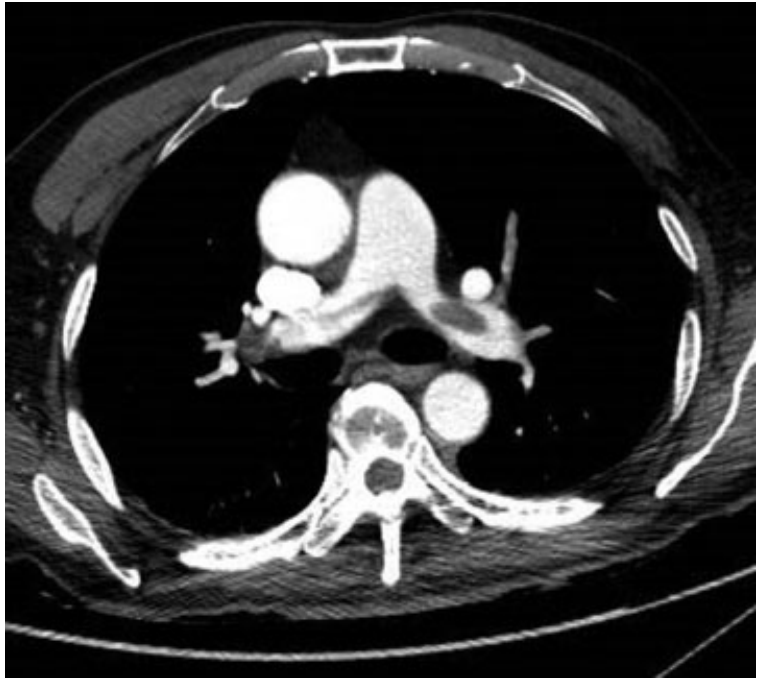

Fig. 3 Computed tomography revealed a nonocclusive thrombus in the left pulmonary artery.

and an asymptomatic PE. Heparin was, thereafter, increased to 15,000 units daily (-Fig. $\mathbf{3}$ ). On postoperative day 21 , the delayed abdominal flap was debrided under local anesthesia. Almost all tendons and muscles of the flexor and extensor compartments, as well as the median nerve, were necrotic. To prevent osteomyelitis and to achieve a functional forearm, we gave priority to reconstruction of the flexor muscle group.

A free functional gracilis muscle flap transfer was thus chosen for the reconstruction of the flexor muscle group. A two-staged operation was performed to avoid a long operative time. On the 35th day after reimplantation, debridement of necrotic tissue and marking of the recipient vessels and motor nerves of the flexor group were performed with heparin using axillary block anesthesia. The operation time was 2 hours 46 minutes and the bleeding volume was $330 \mathrm{~mL}$. Anticoagulation therapy was administered for the PE, but the DVT persisted. A temporary IVC filter was thus implanted on the 39th day after reimplantation (the day before the second operation). On the 40th day, a free gracilis muscle transfer was performed using heparin. We harvested a gracilis muscle flap under general anesthesia, and a sural nerve graft was performed for the reconstruction of the

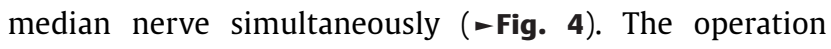
time was 9 hours 45 minutes and the bleeding volume was $645 \mathrm{~mL}$. Two units of red cell concentrates were transfused. The muscle survived and autokinesis of the transferred gracilis muscle was recognized 9 months postoperatively. One year later, the patient suffered from osteomyelitis, and vascularized fibula transplantation was performed for the reconstruction of the radius ( - Fig. 5). In the near future, tendon exfoliation is planned for the flexor side of the digits.

\section{Discussion}

Free muscle transfer is the gold standard procedure for the reconstruction of a functional upper extremity. A neurovas-
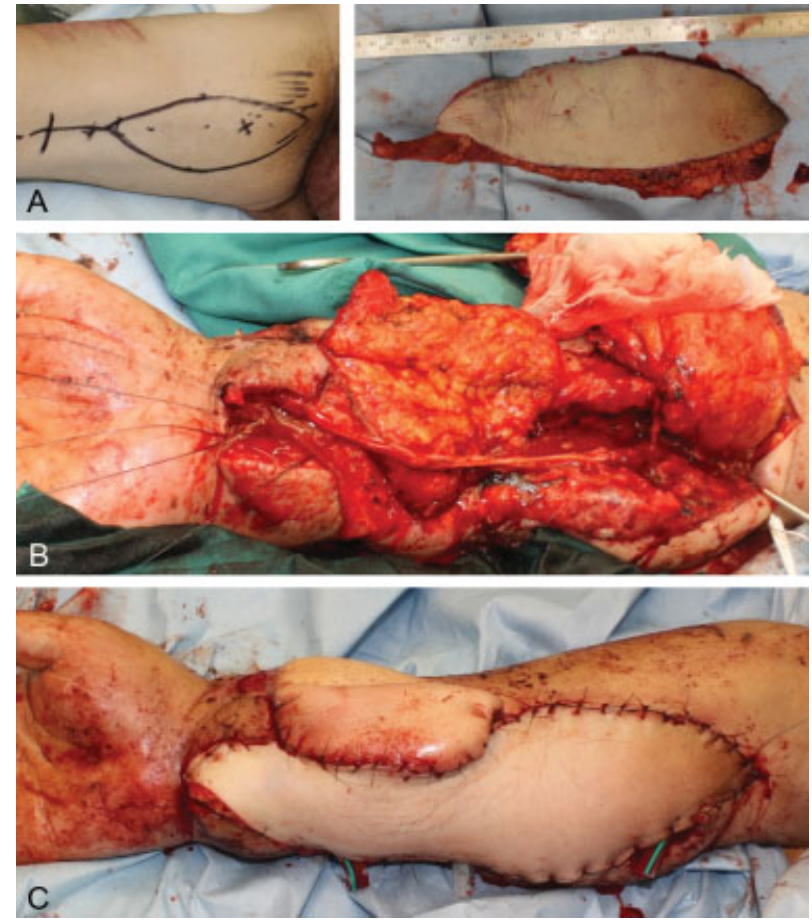

Fig. 4 Free gracilis muscle transfer operation. (A) $7 \times 23 \mathrm{~cm}$ flap was designed inside the thigh and harvested. (B) Harvesting the fat flap to divide the grafted abdominal flap and cover the exposed bone with a fat flap. The median nerve was reconstructed using a cabled sural nerve graft. The distal end of the gracilis muscle was sutured to the flexor digitorum tendon. End to side anastomosis was performed between the flap artery and the radial artery. The vein was anastomosed to the companion vein. The motor nerve of the gracilis muscle was sutured to a branch of the median nerve. (C) Postoperative view.

cular anatomical structure that allows for transplantation, adequate length and excursion, and acceptable donor-site morbidity is necessary for donor muscles. The gracilis, latissimus dorsi, tensor fasciae latae, and medial gastrocnemius, and others can be used as donor muscles. ${ }^{1-4}$ Of these, the gracilis muscle is one of the most commonly used for the reconstruction of the flexor group because the gracilis muscle has a parallel muscle configuration, adequate excursion, a reliable pedicle, and less donor-site morbidity. ${ }^{1-4}$
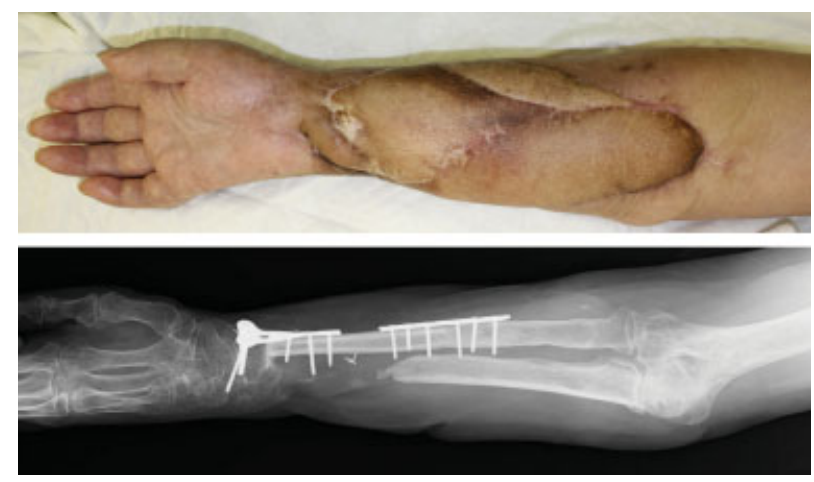

Fig. 5 Nine months after fibular flap reconstruction and X-ray image. 
One of the most important points in the present case is that free gracilis muscle transfer was performed in a patient with known DVT. Because DVT can lead to fatal complications including PE, thoughtful perioperative management is necessary for patients at high risk. ${ }^{6}$ Risk factors for DVT/PE include aging, trauma, malignancy, obesity, orthopedic or abdominal surgery, and prolonged bed rest. ${ }^{5}$ With the presence of lower extremity trauma, including hip fracture as in this case, the risk of developing of DVT/PE becomes very high. ${ }^{10}$ McNamara et al reported that the incidence of symptomatic venous thromboembolism after hip fracture is $2.2 \%{ }^{10}$ Depending on the risk level, DVT prophylaxis includes early ambulation, elastic compression stockings, intermittent pneumatic compression, and chemical prophylaxis. ${ }^{5}$ Regarding the treatment of DVT, it is important to select appropriate medical therapy, catheter-based treatment, or surgical thrombectomy, with careful consideration of the clinical severity and the patient's comorbidities. In this case, while 10,000 units of heparin daily had been administered for chemoprophylaxis after forearm reimplantation, both DVT and PE developed. The heparin dose was thus presumed to be insufficient and was therefore increased before muscle transfer. A more effective strategy may have been to adjust the heparin dose more strictly from the beginning according to the activated partial thromboplastin time value.

Although there are reports of temporary IVC filter placement as a preoperative strategy to manage DVT, there are no clear guidelines. ${ }^{7-9}$ There are many risk factors for venous thromboembolism in free flap operations, including (1) long operative times, (2) positional changes for flap harvest, and (3) suspension of anticoagulant to avoid perioperative bleeding. Even though anticoagulation for DVT/PE was initiated, complete resolution of the thrombus takes time, as it sometimes becomes organized and persists despite therapy. In this case, waiting for the thrombus to resolve on anticoagulation and delaying gracilis transfer could have led to a bad functional outcome for the patient. For that reason, we implemented a treatment strategy to proceed safely with surgery in the presence of a known DVT/PE. Importantly, we divided the surgery into two stages. If an additional surgical team can participate in the surgery, the surgery can safely and expeditiously be performed in a single stage, as the first surgeon can perform preparation of the recipient, while the second surgeon is simultaneously harvesting the muscle. Unfortunately, we could not employ this strategy. Temporary IVC filter was placed preoperatively and anticoagulation therapy (intravenous administration of heparin) was continued intraoperatively as deemed safe. Although the risk of bleeding increased with the continuation of anticoagulation therapy, the use of a tourniquet and suspension of anticoagulation therapy while harvesting the myocutaneous flap minimized blood loss. Using these measures, we were able to successfully complete the free flap reconstruction without a fatal PE or development of a new DVT.

\section{Conclusion}

Lower extremity fracture is a major risk factor for DVT/PE. Despite chemoprophylaxis with heparin, DVT/PE occurred in the present case. There are no established guidelines for reconstructive surgery including free flap in the presence of known DVT/PE. Thoughtful perioperative management to reduce risk factors that exacerbate or incite DVT/PE can help with the safe completion of reconstructive surgery in cases with known venous thromboembolism.

Conflict of Interest

None.

\section{References}

1 Stevanovic M, Sharpe F. Functional free muscle transfer for upper extremity reconstruction. Plast Reconstr Surg 2014;134(02): 257e-274e

2 Fischer JP, Elliott RM, Kozin SH, Levin LS. Free function muscle transfers for upper extremity reconstruction: a review of indications, techniques, and outcomes. J Hand Surg Am 2013;38(12): 2485-2490

3 Yang Y, Yang JT, Fu G, et al. Functioning free gracilis transfer to reconstruct elbow flexion and quality of life in global brachial plexus injured patients. Sci Rep 2016;6:22479

4 Fan C, Jiang P, Fu L, Cai P, Sun L, Zeng B. Functional reconstruction of traumatic loss of flexors in forearm with gastrocnemius myocutaneous flap transfer. Microsurgery 2008;28(01):71-75

5 Kearon C, Akl EA, Comerota AJ, et al. Antithrombotic therapy for VTE disease: antithrombotic therapy and prevention of thrombosis: American College of Chest Physicians evidence-based clinical practice guidelines. Chest 2012;141(2, Suppl):e419S-e496S

6 Klok FA, van Kralingen KW, van Dijk AP, et al. Quality of life in long-term survivors of acute pulmonary embolism. Chest 2010; 138(06):1432-1440

7 Ishihara K, Hiromatsu S, Shintani Y, Kanaya K, Mikasa K, Aoyagi S. Clinical outcome of perioperative nonpermanent vena cava filter placement in patients with deep venous thrombosis or blood stasis of the vein. Surg Today 2009;39(09):764-769

8 Dhand S, Stulberg SD, Puri L, Karp J, Ryu RK, Lewandowski RJ. The role of potentially retrievable inferior vena cava filters in highrisk patients undergoing joint arthroplasty. J Clin Diagn Res 2015; 9(12):TC01-TC03

9 Ryan E, Kok HK, Lee MJ. Retrievable IVC filters - friend or foe. Surgeon 2017;15(02):104-108

10 McNamara I, Sharma A, Prevost T, Parker M. Symptomatic venous thromboembolism following a hip fracture. Acta Orthop 2009;80 (06):687-692 